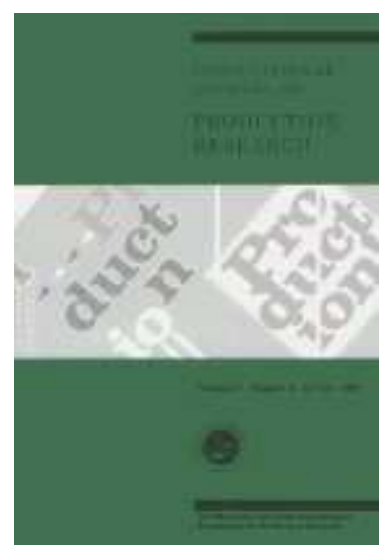

\title{
DESIGN SUPPORT TOOL FOR MATERIAL AND PROCESS COMBINATIONS DURING EARLY DESIGN
}

\begin{tabular}{|r|l|}
\hline Journal: & International Journal of Production Research \\
\hline Manuscript ID: & TPRS-2005-IJPR-0034.R1 \\
\hline Manuscript Type: & State-of-the-Art Review \\
\hline $\begin{array}{r}\text { Date Submitted by the } \\
\text { Author: }\end{array}$ & 07-Oct-2005 \\
\hline Keywords: & $\begin{array}{l}\text { ADVANCED MANUFACTURING TECHNOLOGY, CAD/CAM, DESIGN, } \\
\text { EXPERT SYSTEMS }\end{array}$ \\
\hline Keyword Authors: & $\begin{array}{l}\text { Howard, Lorraine; C.I.T, Mechanical Engineering } \\
\text { Engineering }\end{array}$ \\
\hline Keywiversity of Limerick, Department of Mfg and Ops \\
\hline
\end{tabular}

\section{ScholarONE \\ Manuscript Central}


1

2

3

4

5

6

7

8

9

10

11

12

13

14

15

16

17

18

19

20

21

22

23

24

25

26

27

28

29

30

31

32

33

34

35

36

37

38

39

40

41

42

43

44

45

46

47

48

49

50

51

52

53

54

55

56

57

58

59

60
Name:

Lorraine Howard

Address: Dept. of Mechanical Engineering, Cork Institute of Technology, Bishopstown, Cork. Ireland.

Affiliation: Dr. Huw Lewis, Department of Manufacturing \& Operations Engineering, University of Limerick. Ireland.

Telephone no.: $021-4326742$

Fax no.: $\quad$ 021-4326627

Email: $\quad \underline{\text { howard@cit.ie }}$

Word count: $\quad 4752$
Deleted: 3683 
1. L. Howard, Department of Mechanical \& Manufacturing Engineering, Cork Institute of Technology, Cork.

2. Dr. H. Lewis, Department of Manufacturing \& Operations Engineering, University of Limerick, Limerick.

\begin{abstract}
The aim of this research is to develop an Expert System (ES) that is linked into a 3D design package, which will provide comparison data for various material and manufacturing process combinations to produce a design. The early design stage is the optimum time to ensure that the material and manufacturing process chosen will realise a design's requirements.

This paper focuses on the steps within the process to create the Expert System and provides an example of its use. The CAD package chosen is AutoCAD 2004. A recognition module has been developed to recognise a $3 \mathrm{D}$ model and to segregate the model into that of the quadratic and polyhedron primitives within a database. A generic material and manufacturing database has been designed. This has established a basic platform from which the different material and manufacturing processes can be compared. As each manufacturing process has process specific rules, it is necessary to examine the $3 \mathrm{D}$ model for the suitability of the processing method chosen within the
\end{abstract} ES. 
This ES aims to increase the knowledge available to the designer to aid in the decision-making process, through the comparison reports generated and the web link and specific documentation facility within the software package.

\section{KEYWORDS: CAD, EXPERT SYSTEM, DESIGN}

\section{INTRODUCTION}

Initial design is a vital stage of a successful product's development. It is at this stage that the qualitative and vague marketing definitions of a product are quantified, and as the complexity of the product is increased the more alternatives there are that will match the required characteristics of the product. To find the "Best" or "Optimal" design is an iterative process. Previously, the attention had always been focused on the manufacturing process to reduce the costs. This only represented about $30 \%$ of the products cost area. It has been estimated that design costs only $5 \%$ but that as much as $70 \%$ of the cost of a manufactured part is decided at this stage [1]. Design is an area where a good return on time investment can be realized through good design practice. There are many Concurrent engineering techniques, which prove invaluable to good design practice, utilizing QFD, the Design for suite (DFA, DFS, etc), Axiomatic design and Value engineering, to mention but a few. Yet at the end of all of these techniques one problem remains for the designers, the optimal material and manufacturing process combination.

With a design in hand the designer needs to know what material should be chosen and how can it be processed to suit their specific needs, simultaneous to the 3D design. 
models, such as machining [2-4], casting [5], injection moulding [6], forming [7], etc, and these are viable tools when a process route has been established for a design and finite costing for tooling and fixturing is required, but these do not consider material. There are also material knowledge systems $[8,9,10]$ available that consider material and manufacturing compatibility, which is invaluable at the final stages of design with the in-depth knowledge of materials provided. Yet at the earlier stages in design these systems do not provide comparison charts to depict the associative costs and aesthetics expected for the various material and manufacturing combinations. Nor do they provide details comparing the suitability of different processing methods for a 3D design. This ES aims to fulfill this niche,

The materials available for selection, are extensive, as are the methods of manufacture to produce a design. Not all materials lend themselves to the same method of manufacture and each manufacturing method has its specific design rules. It is within this discipline that this research focuses. The ES developed provides a designer with the data required to screen and select a suite of suitable materials for the design. Each material can be investigated to compare the different manufacturing processes suitable. Manufacturing difficulties within the design are then informed to the designer through the ES. On completion of the ES the designer is presented with reports detailing the suitability of the design for each manufacturing process considered for the material, along with a breakdown of the range of costs, economic order quantity (EOQ), and aesthetics. With these a design team can compare the attributes of the material and manufacturing process combinations and select the combination that best suits their needs for the design. This information offered simultaneous to 3D design aims to bridge

Deleted: With a design in hand the
designer needs to know what material
should be chosen and how can it be
processed to suit their specific needs.
Deleted: kingdom of
Deleted: s
Deleted: is
Deleted: vast,

Deleted: optimal

Deleted: for 
2

3

4

5

6

7

8

9 the gap between CAD and Computer Aided Process Planning (CAPP) to aid product development and improvement.

The methods used to develop the system will be database design, and expert system development, along with concurrent engineering methodology.

\section{THE ES AND CAD SYSTEM}

The CAD package chosen to establish the ES within was AutoCAD 2004. It has solid model capabilities and also lends itself towards customisation. The ability of the system user to continue working within a familiar environment greatly reduces their learning curve when dealing with new software. The AutoLISP language was chosen to extract the 3D model data initially and Visual Basic Applications (VBA) provides the user-friendly interface and the programming capabilities to create the ES and communicate with the different software used.

\subsection{The 3D model capabilities}

The 3D model is constructed using Boolean operations on basic primitives or swept shapes. Utilising this method ensures the 3D model created will be a valid solid. The 3D model does not require tapers or radii as these only increase computations and these process dependant features will be advised for the process chosen at a later stage in the ES.

The 3D model volume and bounding box detail (the minimum and maximum coordinates) were extracted directly to a text (MPR) file. The information pertaining to the 3D model structure was retrieved from the AutoCAD database in the form of Boundary Representation (B-rep) data. This defines the boundary information of a solid
Deleted: Within AutoCAD t

\begin{tabular}{|l|}
\hline Deleted: by \\
Deleted: the chosen \\
\hline Deleted: $\uparrow$ \\
\hline
\end{tabular}

Deleted: that is

Deleted: is 
in terms of face, edge and vertices, together with the information on how they are connected. The B-Rep data extracted is in a SAT file format.

A SAT file is a 3D format created by Spatial Technologies and is characterised as a 3D boundary representation geometric model, which is supported by many solidmodelling systems. The SAT file is the basic file required from AutoCAD for conversion to the STEP format. There has been some standardisation within the $\mathrm{CAD} / \mathrm{CAM}$ industry to facilitate the integration of products, through the use of STEPS [11]. This has established a common platform from which software packages can compare and assign to their particular tasks. STEP conversion is a third party software [12] and is also still in its infancy, so it was not feasible to incorporate it into this stand alone ES. Thus the need to use the SAT file, The main drawback of the SAT file was the interpretation of data for the 3D model. The geometric data associated with the BRep model was obvious but the topology of the model was not clearly evident within the SAT file and this would require the development of algorithms to determine the connectivity of the edges and planes within the 3D model.

Algorithms were developed to extract the geometric data from the text file into lists denoting the entities present within the B-Rep model. Mathematical models were then developed to examine the relationships between the entities to determine existence of the basic polyhedron and quadratic primitives. These models provided the logic for the algorithms used to verify the existence of quadratic and polyhedron primitives occurring within the model. Papers detailing this procedure have been written $\lceil 13,14\rceil$.

\section{An ES database was created to store information on the 3D CAD model data} extracted. The information transferred to the database was of the following format:

\section{Quadratic primitives: A table which denotes circular data regarding:}

Deleted: This is achieved through AutoLISP using the ASCIOUT command, which produces a SAT file of data strings [2-3].

Deleted: 9

Deleted: was retrieved,

Formatted: Numbered + Level: $1+$ Numbering Style: $1,2,3, \ldots+$ Start at: $1+$ Alignment: Left + Aligned at: $28.35 \mathrm{pt}+$ Tab after: $46.35 \mathrm{pt}+$ Indent at: $46.35 \mathrm{pt}$ 
a. The acting axis of a cylindrical feature.

b. The central co-ordinates of this axis.

c. Whether it's a full or partial radius.

d. The number of cylindrical features on the same axis.

e. The minimum and maximum depth of the feature.

f. The minimum radius (if a taper exists) and the maximum radius.

g. Blind or through feature.

h. External boss or not.

\section{Polyhedron primitives: Consisting of four tables}

a. Containing the plane detail and the associated edge quantity.

b. Containing Edge detail and a link to plane identity (above).

c. Containing Edge detail with a unique edge identity.

\section{d. Containing the Point detail.}

\section{Mass properties : A table containing the volume of the 3D model, along with}

\section{the bounding box coordinates of the 3D model.}

The designer initiates the ES (Fig 1), by clicking on the design icon prompting, the designer to select the 3D model. Once this has been done the data concerning the model is extracted to the pre-designed database for the 3D Model. Further computation of the 3D data occurs later in the ES. At this stage in the process the designer is presented with selecting the property profile required by the 3D model (Fig 1). These are the material and manufacturing modules of the $\mathrm{ES}_{-\mathrm{m}}$
Formatted: Numbered + Level: $2+$ Numbering Style: $a, b, c, \ldots+$ Start at: 1 + Alignment: Left + Aligned at: $64.35 \mathrm{pt}+\mathrm{Tab}$ after: $82.35 \mathrm{pt}+$ Indent at: $82.35 \mathrm{pt}$

Formatted: Bullets and Numbering
Formatted: Numbered + Level: $2+$ Numbering Style: $a, b, c, \ldots+$ Start at: 1 + Alignment: Left + Aligned at: $64.35 \mathrm{pt}+$ Tab after: $82.35 \mathrm{pt}+$ Indent at: $82.35 \mathrm{pt}$

\section{Deleted:}

Formatted: Font: Not Bold, Not Italic

Formatted: Bullets and Numbering Formatted: Font: Not Bold, Not Italic

Formatted: Indent: Left: $28.35 \mathrm{pt}$ Hanging: $28.35 \mathrm{pt}$

Deleted: The geometric data associated with the model was transferred to the database. As the topological (i.e.,

adjacency relations) information is not readably retrievable from the SAT file through AutoLISP, the connectivity was determined at a later stage in the ES through VBA in order to fully define the polyhedron and quadratic primitives that exist within the 3D model. Within the AutoCAD environment $t$

Deleted: can

Deleted:

Deleted: This

Deleted: s

Deleted: user

Deleted: through AutoLISP and saved Deleted: a

Deleted: Microsoft Access

Deleted: through VBA

Deleted: as B-rep data

Deleted:

Deleted: which are programmed through VBA. 


\section{MATERIAL AND MANUFACTURING PROCESS KNOWLEDGE}

The nature of data needed in the early stages of design differs greatly in its level of precision and breadth from that needed at the embodiment stage. At the early stage the designer requires approximate property values and the widest possible range of materials [15]. For example, for materials such as mild steel or aluminium, ranges are given to cover the properties for the classes within this material. Similarly generic data has been used for the manufacturing processes. A database with both the material and manufacturing tables has been created for the ES, utilising several sources of valid information [8-16-20].

Within this database the basic properties for each material were grouped into tables detialing mechanical, thermal, electrical and environmental attributes. The basic properties of the manufacturing process were grouped in tables for process attributes, size and dimensional accuracy, economics, model capabilities and material suitability.

When selecting a material for a design the following factors need to be decided on by the design team [15]:

- What properties are required and whether the required properties will be maintained during the service life of the product.

- Whether it will cause environmental problems.

- Whether the material can be processed to the required shape and dimensional accuracy.

- Whether the material can be economically used to produce the product.

The first two factors are addressed within the material selection module and the latter two within the manufacturing process module within the ES. 


\subsection{Material selection module}

A designer decides what performance is needed by a design, through establishing a property profile. This is defined by the function, objective and constraints required by the design. A design may have one or more functions. Its function may be to transmit heat, to support a load or to contain pressure for example. As well as this the design must fulfil an objective. It may be required to be as lightweight and / or as cheap as possible. All designs have constraints, which could be the environment, temperature range or the load it must withstand. All these criteria create a property profile for the design. By applying these property limits the wide range of materials is narrowed through screening. This may appear over simplistic, but the material selection process must be broken down into clear concise steps to facilitate programming.

The ES initially requires the designer to input the required property profile through the mechanical and/or thermal/electrical profile options (Fig1). The property profile can be input as a minimum or maximum value required . Note that only values larger than zero are considered by the ES. The designer is then required to input the level of environmental protection required by the design (Fig 2). Utilising all these properties, the Microsoft Access query facility screens the database for a suitable subset of materials presented as a drop down list to the designer. Information is the key and the designer is able to view the properties of each material within this subset and print off the data if required. An example for the information available on the mechanical data is shown in Figure 3 for the aluminium alloy family of materials, suitable to the property profile input.

\begin{tabular}{l} 
Deleted: example is shown here for a \\
3D model where the mechanical property \\
inputs are for strength and density (Fig 2) \\
Deleted: The property profile input by \\
the designer is saved to the database. \\
Deleted: \\
\hline Deleted: \\
\hline Deleted: through \\
Deleted: is screened \\
Deleted: . \\
Deleted: This subset of suitable \\
materials is \\
Deleted: environmental
\end{tabular}

\section{.}


The designer can review as many materials as required here, but a single material must be selected to process further. The other materials within the subset can be returned to at a later stage to process.

Within this part of the ES the designer also has the option to link to the Internet for information on materials and their properties, through pre-established web sites. Here the designer can view material selection charts if required. There is also the opportunity, if the ES is being used for a quick review, to choose a material directly from the database to process.

Within the material selection of the ES the option also exists to examine utilising heat treatment to provide the required mechanical properties and/or to examine applying a surface coating to provide the required protection. These are processes, but are important to consider here as they may affect material choice. Once the material choice is finalised the ES proceeds to the manufacturing selection module.

\subsection{Manufacturing process selection module}

The processes that are suitable to the material chosen are screened, The designer is given these processes in a drop down list and can choose to review them individually. Like the material module the attributes associated with the process are available to the designer to view and to print if required. The designer must then choose a process to examine within the ES. It is at this stage that the further computation of the 3D model data occurs.

\subsection{Generating the 3D model specifics}

It is within this section that the real investigation of what the $3 \mathrm{D}$ model contains takes place. The area of feature recognition is vast and this thesis is only touching on the
Deleted: Once the material is chosen this is downloaded to the database

\begin{tabular}{|l|} 
Deleted: and \\
\hline Deleted: in \\
\hline
\end{tabular}

Deleted: through the Microsoft Access query facility

Deleted: through VBA programming 
tip of the iceberg with basic recognition. The reasoning being that this thesis is about an area other that feature recognition alone, but as it encompasses it, it is necessary to achieve some level to proceed.

When integrating CAD with manufacturing applications the difficulty lies in how to interpret the low level CAD information automatically into a useful format for $\underline{\text { selecting an appropriate method of manufacture. Here automatic feature recognition has }}$ been used, where the data structure of the geometric model is examined to discover and extract its features automatically. The mechanism used in the ES to determine automatic feature recognition is a combination of rule-based method, mathematical modelling and graph based methods. This was achieved in Section 2.1, through extracting the data pertaining to the polyhedron and quadratic primitives into the ES 3D database. In order to determine what further feature recognition is required, it was necessary to establish the process parameters required to determine the overall model suitability. The parameters selected were the suitability of

1. The mass of the model,

2. Its overall maximum dimension,

3. The material thickness, and

4. The hole size range allowed

by the process. The parameters for the mass, the maximum dimension and the hole size were readily available in the ES 3D database (Section 2.1). The material thickness required further recognition algorithms to be developed.

In order to establish this parameter, the topology of the model was determined. Firstly the edge connectivity was determined for each plane in the model. Each plane within the 3D model can be bounded by straight edges, angled edges and/or curves. 
Where each edge or curve finishes and another one begins, is connected by a point. This connectivity eventually reaches back where it started from, forming a continuous loop around the shape. Search algorithms were developed using IF-THEN logic to establish this connectivity, where each plane has its edges $\&$ points identified with a unique Loop identity number for each loop on a plane. If more than one loop was found on a plane the loop with the largest internal area was denoted as an external loop and the other as an internal loop.

With these loops identified the next section of programming established the connectivity of the planes within the model. It did so by starting with a plane having an internal loop. Using this loops identity and its edge connectivity, the planes connected to these edges were determined through basic algorithms and the database query facilities. This data was then compared to the other loop identities and edge connectivity's. This search process continues until all the internal and external loops have been accounted for. The resulting table contains data regarding the top and base plane for each non round feature, a link to the loop identities and the overall depth of the feature. With these basic building blocks of the model established, the material thickness can now be determined.

Each internal loop was reviewed to calculate the material thickness. Using each internal loop identity and the building block it belonged to, its relationship with the external loop on the same plane was examined. If the internal loop building block depth was in the same direction as that of the external loops building block depth then the internal loop was an internal feature and the material thickness for that plane was established. If the opposite occurs then the material section has narrowed but, because it was travelling in the opposite direction then it is part of the outer model. Its material

\section{Deleted: At the end of this module}

Deleted: With the loops established it is now possible to set up VBA subroutines to determine specific details about the $3 \mathrm{D}$ model which is needed for the following sections on the determination of process suitability and specifics. 9

Firstly, utilising the loops on a plane enabled the calculation of the distances

between the outside of a circular feature (hole or boss) touching that plane and an outer edge or inner feature. The minimum distance calculated from each loop on a plane is downloaded to the database for each face of a circular feature. . Secondly,

Deleted: seeks to 
thickness will be picked up by the next internal loop searched. If there are no internal sections then the overall thickness of the model along the $\mathrm{Z}$ axis was considered as the material thickness,

Another process rule that occurs regularly is the nearness of a feature to an edge.

Utilising the knowledge developed about the loops on each plane enabled the calculation of the distances between the outside of a circular feature (hole or boss) touching that plane and an outer edge or inner feature. The minimum distance calculated from each loop on a plane was downloaded to the database for each face of a circular feature.

The database now had enough information about the 3D model to proceed in examining the processes suitability and model specifics.

\subsection{The 3D model process suitability}

The overall suitability of this process for creating the $3 \mathrm{D}$ model is reviewed through comparison of the information generated through the recognition algorithms and that from the relevant tables within the database. The suitability is then informed to the designer through the ES (Fig 4).

Even if the process is unsuitable for any of the above the designer can still proceed to examine the process and generate the reports. Remember design is an iterative process and the economics report may encourage redesign of the model at this early stage. Also as the database is generic it contains ranges of data, which can over exaggerate the figures, for example the allowable mass, whereas when a specific material type is chosen this may be found to be suitable.

So far only the basic suitability of the process has been considered. Every process has manufacturing rules, which need to be adhered to. These are rules like hole depth to

Deleted: non-round internal features within the 3D model. It looks for basic slots or hollows within the 3D model, as well as the outside basic shape. It does so by starting with an internal loop on a plane and determining its connectivity. When all the internal loops have been accounted for, the external loops without prior connectivity are accounted for. This is necessary to account for internal features and also to determine the material thicknesses of the model.
Deleted: has

\begin{tabular}{l}
\hline Deleted: \\
\hline Deleted: through \\
\hline Deleted: 9 \\
$<\#>$ The suitability of the mass of the \\
model, $\uparrow$ \\
$<\#>$ Its overall maximum dimension, $\uparrow$ \\
$<\#>$ The material thickness and \\
$<\#>$ The hole size range allowed by the \\
process. \\
This \\
\hline Deleted: is retrieved from \\
Deleted: and compared
\end{tabular}

Deleted:

\section{Deleted: 9}

$<\#>$ The suitability of the mass of the

Deleted: and compared 
diameter ratios, suitability of the different axes for processing features, minimum radii, and so on. This process information set up as a table within the ES material database. These process rules are advised now rather than at a later stage in design, when all the detailed drawings have been finalised and the model is sent to the manufacturer for exact costing. The design at this stage is still in its infancy and as accurate costing will eventually need to be sought, it would be beneficial not to have to redesign at a later stage. So these rules are considered now.

\subsection{The 3D model processing specifics}

The option to review the specifics of the 3D model with respect to the chosen process is given to the designer (Fig 4). The specifics reviewed are those, which apply to the bosses, the round and non-round holes within the model. For example, for each hole within the model, the suitability of its axis for processing, the hole type - through or blind, the diameter to depth ratio and its distance from each edge on a plane is checked through comparison algorithms within the ES database. In this example, the $22 \mathrm{~mm}$ deep through hole, $10 \mathrm{~mm}$ in diameter (Fig 1) is reviewed. For the Die press and sintering process selected, the depth to diameter ratio for a through hole is $4: 1$, the edge distance of the hole from its nearest edge is greater than the minimum material thickness of $1.5 \mathrm{~mm}$ allowable between holes and edges. This $3 \mathrm{D}$ model is shown to be within these specifications. Yet the axis along which the hole is to be processed is not allowable by Die press and sintering. The designer is informed here that a secondary operation is required (Fig 5). Similarly, the boss and the internal slot (Fig 6) show up as requiring secondary operations to create. Therefore the designer would see that this is not a suitable process to proceed with for the design. 


\section{COMPARISON REPORTS}

When content with the processes selected for comparison for a specific material the designer can print off two main reports. The first of these is that of the basic suitability of the process (advised in Fig 4) along with the material chosen and its cost

per Kilogram. The second report (Fig 7 ), details the economics of each process along with their aesthetic ability. This information provides a good basis for comparison between the different processes for a designer.

The cost index is a major economic comparison indicator between the processes. It represents the total cost range per unit to the company. This is based on the material, dedicated tooling and gross overhead costs. The material cost uses the 3D model volume, material density range, material cost and material utilisation from the database for each process. The tooling cost and gross overhead cost are based solely on process economics. 
The aesthetic ability of the process looks at the quality, roughness and tolerances achievable through each process. These would be of vital importance. Secondary operations may be required to achieve the aesthetics of the design. These are not considered by the ES but, by highlighting the aesthetic ability the designer is aware of the capabilities of each process.

In this example, the 'Manufacturing process economic data' report (Fig 6) has shown the comparison data for two near net shape processes for the aluminium alloy $3 \mathrm{D}$ model. With these the designer can see at a glance that if the economic order quantity (EOQ) required was greater than four thousand then the high pressure die casting would be the process to choose. If the EOQ was less than a thousand then the ceramic mould casting would be the chosen process. In this instance the mass of the $3 \mathrm{D}$ model was the main restricting variable for the number of suitable processes, coupled by the process specifics. The former could be rectified by a material change. Following the completion of the above reports the designer can review another material from the original screened set of materials to investigate the processing of, if so required. This is in keeping with the iterative nature of design as there is no one single solution and knowledge is the key to ensuring the best design is chosen.

\section{CONCLUSION}

During the design, the material and manufacturing process combinations will be chosen from those with which the product design team is most comfortable [1]. As a result, the opportunity for major manufacturing improvements may be lost through the limited selection of manufacturing processes and associated materials in the early stage of product design. This ES would provide a useful tool when designing in an era where 
The aim here was to create an ES that would be simple to use and could supply knowledge in the various areas required for product design. At the early stages of design this ES could be utilised easily by a design team. The ES works within a familiar environment and provides the designer with a user-friendly graphical user interface (GUI) through simple forms, input boxes, option and command buttons.

This paper has detailed the basic format of the ES created to aid the designer in selecting material and manufacturing process combinations at design. It has shown how the reports and information generated through the ES can aid the designers with their optimal design combination in an aim to reduce the errors that can appear in designs once they enter the production process. 


\section{REFERENCES}

1. Ullman, D.G., The Mechanical Design Process, 2nd Ed, Mc Graw-Hill, 1997.

2. Licom Internet site. Available on line at : www.licom.com

3. AUTO-CODE Mechanical internet site. Available on line at :

www.autocode.com

4. Intercim NC solutions centre. Available on line at :, www.austinnc.com

5. CAStech software. Available on line at: www.castech.fi

6. Moldflow internet site. Available on line at: www.moldflow.com

7. FemForm software internet site. Available on line at: www.femengineering.com

8. Cebon, D., Ashby, M.F., Lee-Shothaman, L., Cambridge Engineering Selector Version 4 (CES4), 2002, Granta Design Ltd.

9. Material data. Available on line at: www.matdata.net

10. ASM handbook. Available on line at: www.asminternational.org

11. Han, J.H., Kang, M. and Choi, H., STEP-based feature recognition for manufacturing cost optimisation, Journal of Computer-Aided Design, 2001, 33(9), 671-686.

12. STEP conversion from StepTools. Available on line at: www.steptools.com

13. Howard, L., Lewis, H., A manufacturing cost analysis tool for 3D design, 12th International conference Flexible Automation \& Inteligent Manufacturing Dresden, 15-17th July, 2002 pp. 1174-1184. 
14. Howard. L., Lewis. H., 3D feature recognition - an integral part of modern design, $20^{\text {th }}$ International Manufacturing Conference CIT Cork, 3$5^{\text {th }}$ Sept, 2003 pp. 77-83.

15. Ashby, M.F., Materials selection in mechanical design, second edition, 2003, pub Butterworth-Heinemann.

16. Philip, M., Bolton, B., Technology of Engineering Materials, 2002, pub Butterworth-Heinemann.

17. Swift, K.G., Booker, J.D., Process selection from design to manufacture, 1997, pub Arnold.

18. Cubberly, W.H., Bakerjian, R. (Editors), Tool and Manufacturing Engineers Handbook (TMEH), Desk Edition, 1989, pub SME.

19. Beitz, W., Küttner, K.-H. (Editors), Dubbel Handbook of Mechanical Engineering, 1994, pub Springer-Verlag London limited.

20. Boothroyd, G., Dewhurst, P., Knight, W., Product Design for Manufacture and Assembly, Marcel Dekker, Inc., 1994.
Deleted: $<\#>$ Han, J.H., Kang, M. and Choi, H., STEP-based feature recognition for manufacturing cost optimisation, Journal of Computer-Aided Design, 2001, 33(9), 671-686.

Deleted: <\#>Cebon, D., Ashby, M.F., Lee-Shothaman, L., Cambridge Engineering Selector Version 4 (CES4), 2002, Granta Design Ltd. 


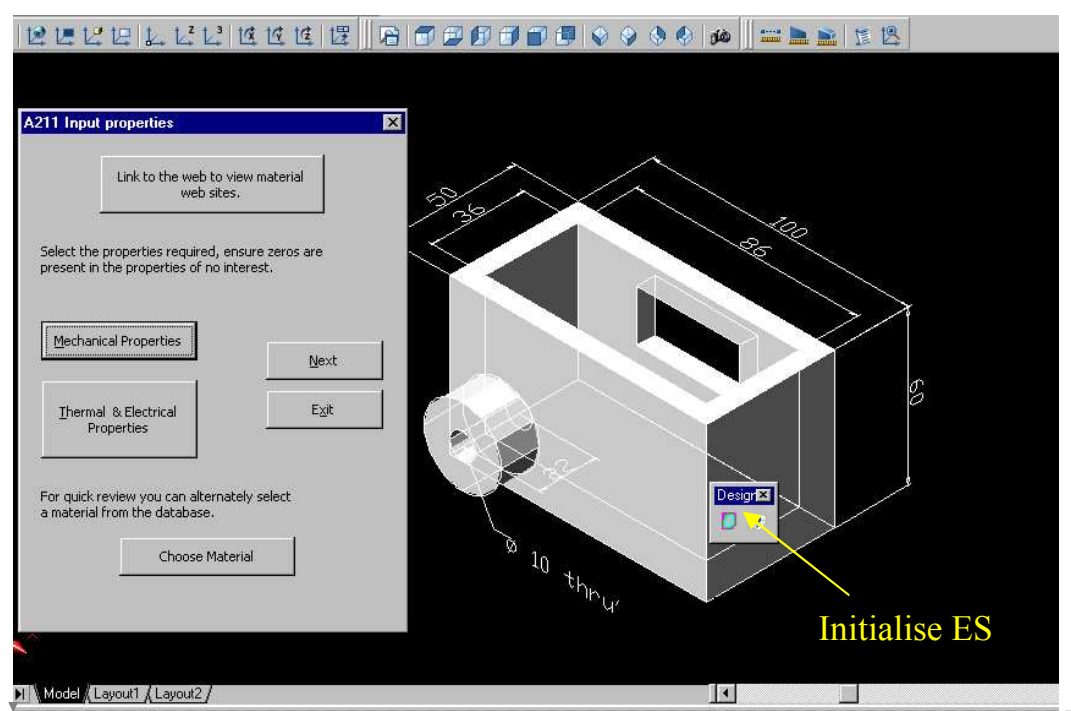

Fig 1. Initialise ES \& select required property profile

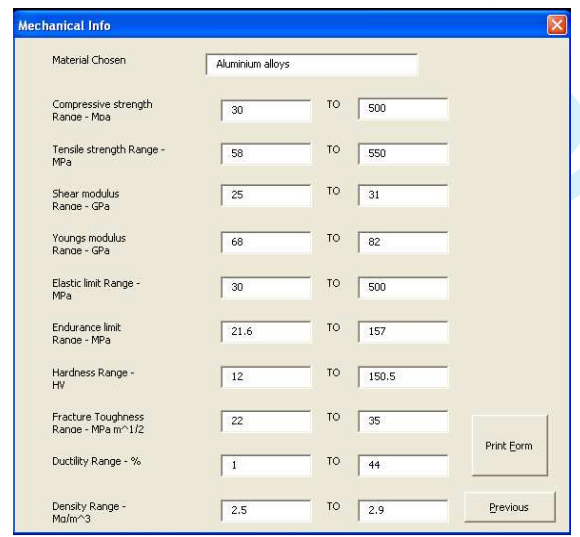

Fig 3. Mechanical properties

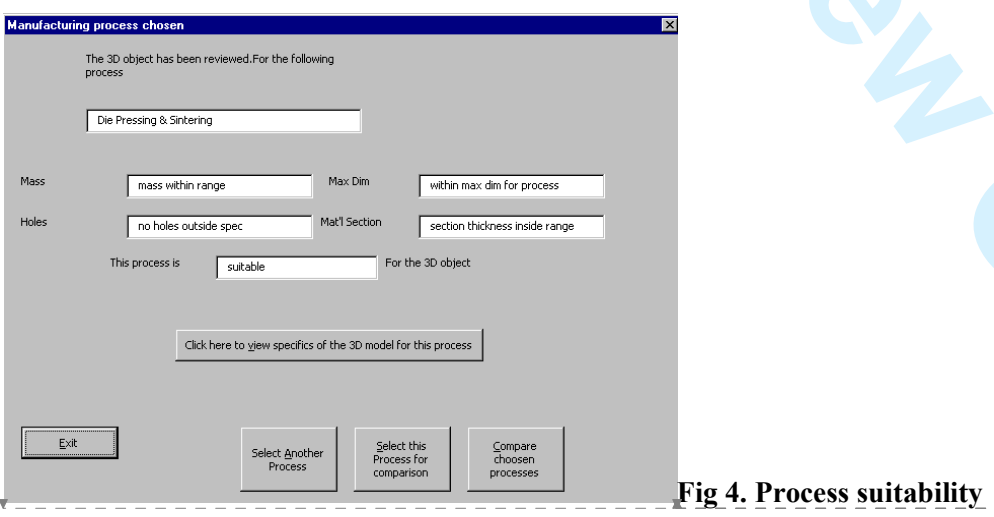

Formatted: Font: $8 \mathrm{pt}$

Formatted: picture

Deleted:

Page Break

Deleted: $<$ sp $>$

Formatted: Font: $12 \mathrm{pt}$, English (Ireland), Check spelling and grammar

Formatted: picture

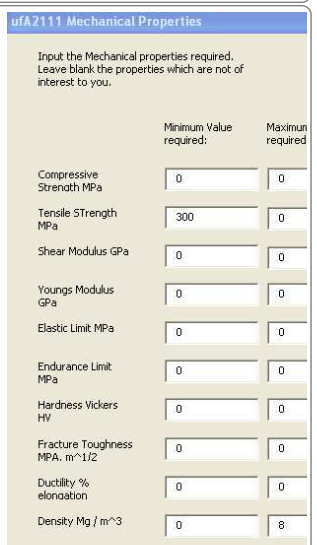

Deleted:

Formatted: Indent: Left: $22.5 \mathrm{pt}$, First line: $7.5 \mathrm{pt}$

Deleted: 2

Deleted:

Deleted: 3

Deleted: 9

Formatted: picture

Deleted: 9

Formatted: Font: 10 pt, Bold

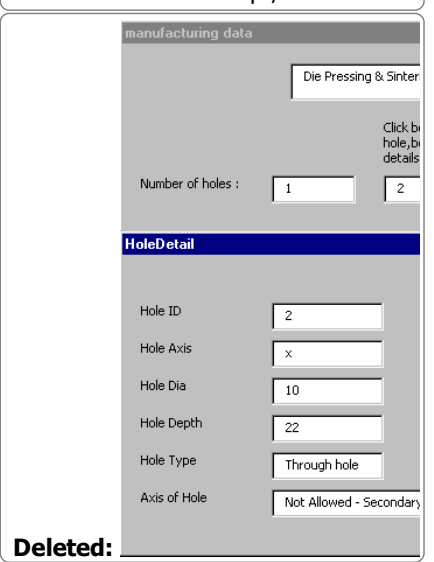




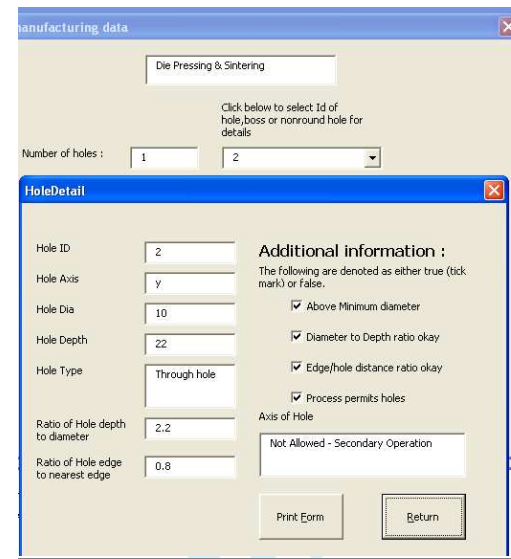

Fig 5. Process specifics for hole
Formatted: Tabs: $213.75 \mathrm{pt}$, Left + Not at $296.25 \mathrm{pt}$

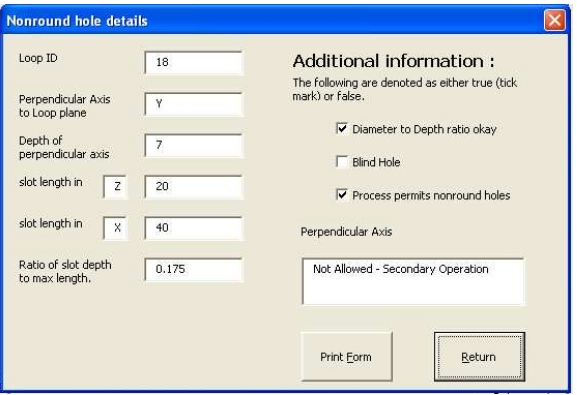

Fig 6. Process specifics for nonround hole
Formatted: Tabs: $217.5 \mathrm{pt}$, Left + Not at 296.25 pt

Formatted: picture

\section{Manufacturing Process Economic Data}

\begin{tabular}{|c|c|c|c|c|c|c|c|c|}
\hline Process Nome & \multicolumn{7}{|c|}{ Ceranic Mould Casting } & \\
\hline Cost Index & \multicolumn{2}{|c|}{ Tooling Cost range } & \multicolumn{2}{|c|}{ Capital range } & \multicolumn{2}{|c|}{ Roughness range (micro $m$ ) } & \multicolumn{2}{|c|}{ Quality range } \\
\hline$€ 18.34 \quad \operatorname{es} .19$ & $€ 139$ & $E 1,394$ & $E 69 \pi$ & $€ 6970$ & 15 & 32 & 1 & 6 \\
\hline$M x E O Q$ & \multicolumn{2}{|c|}{ Lead Time (whs) } & \multicolumn{2}{|c|}{ Material Utilisation } & \multicolumn{2}{|c|}{ Praduction Rate $\left(K_{g} / \mathrm{H} v\right)$} & \multicolumn{2}{|c|}{ Tolerance range $(\mathrm{mm})$} \\
\hline 100 & 1 & 3 & 0.5 & 0.75 & 3 & $\boldsymbol{\pi}$ & $4^{0.38}$ & 08 \\
\hline
\end{tabular}

Process Name High Pressure Die Casting

\begin{tabular}{|c|c|c|c|c|}
\hline Cost Index & Tooling Cost range & Capital range & Roughness range (micro $m$ ) & Quality range \\
\hline E10.70 E9r.77 & $\operatorname{Eg}, 510$ & $\oplus 9 r, \mathbf{m}$ & 08 & 1 \\
\hline $\operatorname{MrxEOQ}$ & Lead Time (wiks) & Material Utilisation & Production Rate $(\mathrm{Kg} / \mathrm{Hr})$ & Tolerance range $(\mathrm{mm})$ \\
\hline 4 8 का & 4 & 0.75 & 40 & 0.15 \\
\hline
\end{tabular}

Fig 7. Economic and aesthetics report

Formatted: Indent: Left: $30 \mathrm{pt}$

Deleted: 6 